and who exhibit stereotyped movements, speech and language disorders, tics, and motor clumsiness. Asperger's disorder may overlap or occur concurrently with Tourette syndrome, pervasive developmental disorder, and attentional deficit disorders.

\title{
DEVELOPMENTAL DYSCALCULIA
}

An 11-year-old, Hebrew speaking boy of normal intelligence, referred to Alyn Hospital, and Schneider Children's Medical Center, Jerusalem, for evaluation of learning and attentional problems, was found to have a profound dyscalculia based on a proposed lack of "cardinal/ordinal skills acquisition device" (COSAD). Several male family members had dysgraphia, right-left disorientation, and dyslexia. At birth, the child was hypotonic, and motor development was delayed, walking independently at 2 and $1 / 2$ years. Neurologic abnormalities included high-pitched voice, dysgraphia, right-left disorientation, finger agnosia, clumsiness in running and jumping, scoliosis, and fine motor incoordination. At 4 years, he developed grand mal seizures treated with carbamazepine, and at 7 years he received pemoline (Cylert) for ADD without hyperactivity. The pemoline benefited overall functioning. The use of linguistic, visual and verbal memory cues compensated for deficits in ordinal number use, but not for cardinal number skills, which remained limited. He could count small numbers, but could not do simple calculations, a skill requiring an innate experience of quantity, less amenable to language, visual-spatial, or logical mediation. Different theories of number development and mathematical skills are discussed in relation to mechanisms of dyscalculia and its management. (Ta'ir J, Brezner A, Ariel R. Profound developmental dyscalculia: evidence for a cardinal/ordinal skills acquisition device. Brain and Cognition November 1997;35:184-206). (Reprints: Judy Ta'ir, Alyn Hospital, PO Box 9117, Jerusalem 91090, Israel).

COMMENT. A child with ADHD and learning disability involving mathematical skills should be examined neurologically for Gerstmann's syndrome (dyscalculia, dysgraphia, right-left disorientation, and finger agnosia). In adults with stroke or cerebral tumor, GS is usually associated with lesions localized to the angular gyrus and parietal lobe of the dominant hemisphere. In children, partial Gerstmann syndromes may be developmental or acquired and may occur with focal lesions in other areas of the dominant hemisphere or with diffuse involvement of either or both hemispheres. An EEG may help in localizing a lesion in children with ADHD, GS, and seizures, and may prompt the need for an MRI.

Profound developmental dyscalculia may occur in children of normal or even superior IQ Deficits in specific cognitive areas may involve visualspatial perception and parietal-occipital dysfunction. In a theoretical hypothesis of development dyscalculia, an innate, highly specific cognitive domain is involved; ordinal number tasks and counting small magnitudes may be successfully completed, whereas larger quantities involving calculations are not possible.

\section{CHILDHOOD ADHD AND ADULT CRIMINALITY}

The relationship between attention deficit hyperactivity disorder in childhood and criminality in adolescence and adulthood was examined in 89 hyperactive and 87 normal control subjects studied prospectively at the Division of Child Psychiatry, Oregon Health Sciences University, Portland, OR. Hyperactive subjects had significantly higher juvenile and adult rates of 
arrest and incarceration compared to controls. Comorbid conduct problems during childhood and serious antisocial behavior in adolescence predicted later criminality in adulthood. (Satterfield JH, Schell A. A prospective study of hyperactive boys with conduct problems and normal boys: adolescent and adult criminality. I Am Acad Child Adolesc Psychiatry December 1997;36:17261735). (Reprints: Dr Anne Schell, Psychology Department, Occidental College, 1600 Campus Rd, Los Angeles, CA 90041).

COMMENT. Hyperactive children are at an increased risk for criminal behavior and arrest in adolescence and in adulthood. Childhood conduct problems and adolescent antisocial behavior are predictors of later arrest for criminality. Hyperactive children have a five times normal rate of developing conduct disorders and a subsequent increased risk of criminality. Stealing from other children, and telling lies to get out of trouble are conduct problems that appear minor but may lead to more serious antisocial behavior in adolescence unless treated by early intervention counselling. Multimodal treatment of ADHD in childhood results in improved academic achievement and less antisocial behavior compared to drug-only treated patients.

\section{HEADACHE DISORDERS}

\section{SUMATRIPTAN NASAL SPRAY FOR ACUTE MIGRAINE}

The efficacy and side effects of intranasal sumatriptan (20 mg, $10 \mathrm{mg}$ ) or placebo in the treatment of a single migraine attack were evaluated in two randomized, double-blind, multicenter studies of 409 and 436 adult patients, funded by Glaxo Wellcome. Pain relief to mild or none occurred at 2-hours in two thirds of patients treated with $20 \mathrm{mg}$ doses, in one half the group receiving $10 \mathrm{mg}$, and in one third of placebo-treated patients. A second dose was given within 24 hours to one-third of patients who had a recurrence of headache. Bitter or unpleasant taste in $25 \%$ was the most common side effect. (Ryan R, Elkind A, Baker CC et al. Sumatriptan nasal spray for the acute treatment of migraine. Results of two clinical studies. Neurology Nov 1997;49:1225-1230). (Reprints: Dr Robert Ryan, 621 South New Ballas Rd, Suite 537, St Louis, MO 63141).

COMMENT. Sumatriptan nasal spray $(20 \mathrm{mg}, 10 \mathrm{mg}$ ) is an effective treatment of acute migraine attacks, and should offer a more convenient and practical means of treatment in migraine-associated vomiting or in patients who object to injections.

Zolmitriptan (Zomig) for acute migraine was found effective in a multicenter, double-blind, placebo-controlled study of 1000 patients. The optimal initial dose was $2.5 \mathrm{mg}$ orally. (Rapoport AM, Ramadan NM, Adelman JU, et al. Neurology Nov 1997;49:1210-1218). In a further trial of $2.5 \mathrm{mg}$ oral dose of zolmitriptan cf placebo in 327 patients, 12 to 65 years old, headache response at 2 hours was $62 \%$ of to $36 \%$ for placebo. (Solomon GD, Cady RK, Klapper JA et al. Neurology Nov 1997;49:1219-1225).

Autosomal dominant familial hemiplegic migraine, previously mapped to $19 \mathrm{p}$ chromosome locus, is linked to chromosome $1 \mathrm{q} 31$ in a 39 member four-generation family from Wyoming. The majority reported minor head trauma as a trigger for attacks. (Gardner K, Barmada MM, Ptacek LJ, Hoffman EP. A new locus for hemiplegic migraine maps to chromosome 1q31. Neurology Nov 1997;49:1231-1238). 